\title{
Laser Interferometer Gravitational-Wave Observatory beam tube component and module leak testing
}

\author{
W. A. Carpenter and P. B. Shaw \\ Chicago Bridge \& Iron Co., Plainfield, Illinois 60544-8929 \\ L. Jones \\ California Institute of Technology, Pasadena, California 91125 \\ R. Weiss \\ Massachusetts Institute of Technology, Cambridge, Massachusetts 02139
}

(Received 1 October 1999; accepted 20 December 1999)

\begin{abstract}
Laser Interferometer Gravitational-Wave Observatory (LIGO) is a joint project of the California Institute of Technology and the Massachusetts Institute of Technology funded by the National Science Foundation. The project is designed to detect gravitational waves from astrophysical sources such as supernova and black holes. The LIGO project constructed observatories at two sites in the U.S. Each site includes two beam tubes (each $4 \mathrm{~km}$ long) joined to form an " $\mathrm{L}$ " shape. The beam tube is a $1.25 \mathrm{~m}$ diam $304 \mathrm{~L}$ stainless steel, ultrahigh vacuum tube that will operate at 1 $\times 10^{-9}$ Torr or better. The beam tube was manufactured using a custom spiral weld tube mill from material processed to reduce the outgassing rate in order to minimize pumping costs. The integrity of the beam tube was assured by helium mass spectrometer leak testing each component of the beam tube system prior to installation. Each $2 \mathrm{~km}$ long, isolatable beam tube module was then leak tested after completion. (c) 2000 American Vacuum Society. [S0734-2101(00)03204-8]
\end{abstract}

\section{INTRODUCTION}

This article discusses the leak detection procedures used to test the $16 \mathrm{~km}$ of $1.25 \mathrm{~m}$ diam ultrahigh vacuum beam tube used in the Laser Interferometer Gravitational-Wave Observatory (LIGO) project. The beam tube was leak tested in four steps including:

(i) leak testing fabricated $20 \mathrm{~m}$ long tube sections,

(ii) local leak testing of $250 \mathrm{~mm}$ diam valves and valve nozzles,

(iii) leak testing circumferential welds joining tube sections together, and

(iv) final leak testing of the installed $2 \mathrm{~km}$ long beam tube modules.

The component leak testing included 800 tube sections (20 m long), 808 circumferential welds, and 72 valved nozzles. Each component was tested to a sensitivity of better than $1 \times 10^{-10} \mathrm{~atm} \mathrm{cc} / \mathrm{s}$ of helium.

The leak test of the $2 \mathrm{~km}$ long beam tube modules would have been extremely difficult and expensive using standard helium leak detection techniques. Therefore, a method was developed utilizing a residual gas analyzer (RGA) to measure the leak tightness of the tube module. Another method was developed to locate leaks from the pressure gradients in the tube using nine RGAs distributed along the length of the tube.

\section{PROJECT BACKGROUND}

The LIGO project's mission is to detect gravitational waves from astrophysical sources such as a supernova or the formation of black holes. Two LIGO sites have been constructed in the U. S., one near Richland, Washington on the
Hanford Reservation and another at Livingston, Louisiana. The initial detectors are currently being installed and commissioned and the entire system is planned to be operating at design sensitivity by 2002 .

At each site the beam tubes have a diameter of $1.25 \mathrm{~m}$ and are configured as an L with $4 \mathrm{~km}$ long arms. The tube along each arm is divided into $2 \mathrm{~km}$ isolatable beam tube modules. The entire project required $16 \mathrm{~km}$ of beam tube. The tubes are covered with an arch shaped concrete cover to protect them from the environment and to reduce their wind driven motion. A view of the Hanford facility is provided in Fig. 1.

The concept of the LIGO detector is to sense the distortions of space induced by incoming gravitational waves with laser interferometry between suspended mirrors (test masses). The test masses are placed in vacuum chambers at the vertex and ends of the L. The gravitational wave will shorten the time it takes light to travel between test masses along one arm of the $\mathrm{L}$ while lengthening the time in the other arm. The difference in travel time along the arms is proportional to the strength of the gravitational wave. Laser light enters the system at the vertex of the $\mathrm{L}$ and is split between the two arms. After traversing the arms multiple times by bouncing between the test masses, the beams are recombined at the vertex to measure the difference in travel time.

The changes in length of the $4 \mathrm{~km}$ arms due to gravitational waves of astrophysical origin are expected to be less than $10^{-17} \mathrm{~m}$ so that significant effort is required to minimize the perturbations on the test masses from seismic and thermally induced (Brownian) motion. In order to sense such small motions, the laser beams must propagate in a good vacuum and the effect of light scattering from the beam tube walls needs to be controlled. The outgassing of the beam 


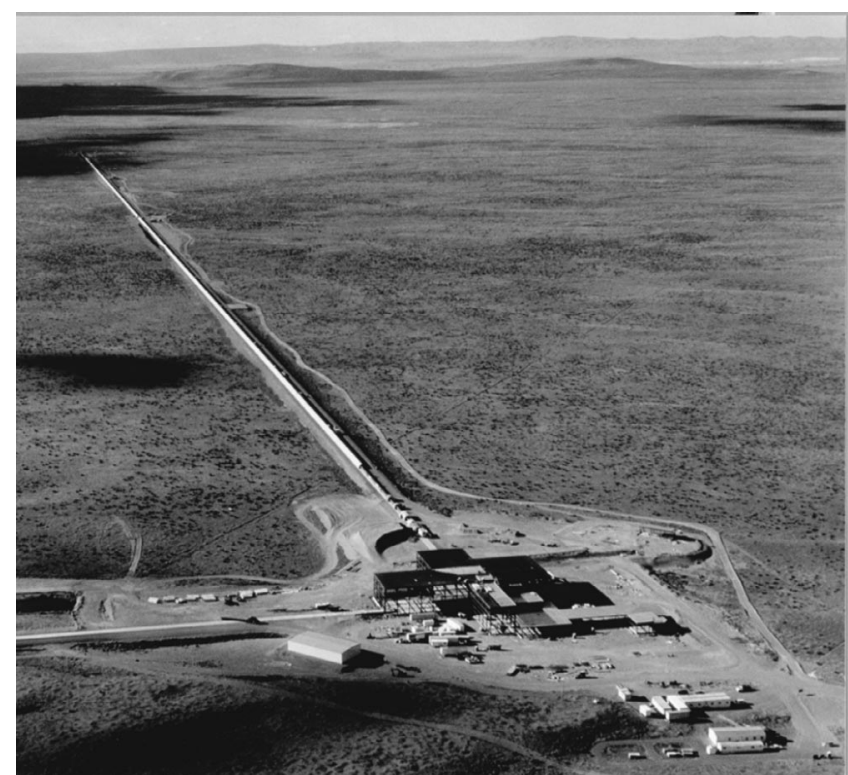

FIG. 1. Aerial view of the LIGO site at Richland, Washington.

tube material and the baffling strategy as well as the surface treatment of the beam tubes is determined by the goal of accommodating future interferometers operating at the quantum limit.

The two sites will operate in coincidence, exploiting the property that a gravitational wave from an astrophysical event will distort the space almost simultaneously at the two sites, while most of the perturbing noise is uncorrelated. The LIGO detectors will comprise one element of a worldwide network of detectors with additional sites in Italy, Germany, Japan and Australia. The network, using the slight variations in the arrival time of the waves over the Earth, will determine the position of the sources in the sky.

The tubes are manufactured of $3 \mathrm{~mm}$ thick, $304 \mathrm{~L}$ stainless steel coils, specially treated to reduce hydrogen outgassing to a rate of $1 \times 10^{-13}$ Torr $\ell / \mathrm{s} \mathrm{cm}^{2}$ or less. During operation, the beam tubes will be evacuated to a partial pressure of $1 \times 10^{-9}$ Torr of hydrogen and $1 / 10$ of this partial pressure for water, carbon monoxide, and carbon dioxide to minimize the propagation time fluctuations due to molecular density fluctuations in the laser beam.

A custom designed tube mill was used to manufacture the specially treated stainless steel coil material into $20 \mathrm{~m}$ long spiral welded tube sections. External stainless steel stiffeners were welded to the beam tube sections at $0.75 \mathrm{~m}$ intervals along the tube length to resist buckling due to the external pressure. A bellows was added to half of the beam tube sections to allow for tube length changes when the system is baked out and to accommodate daily and seasonal temperature variations. About $10 \%$ of the tube sections were also provided with a $250 \mathrm{~mm}$ port and high vacuum valve. The valves are located about every $250 \mathrm{~m}$ along the beam tube modules for diagnostic purposes and as pump ports for the system bakeout. The beam tube sections were then welded at the site into the $2 \mathrm{~km}$ beam tube modules with 44 in. (1120 $\mathrm{mm}$ ) diam high vacuum valves at each module end. A photo

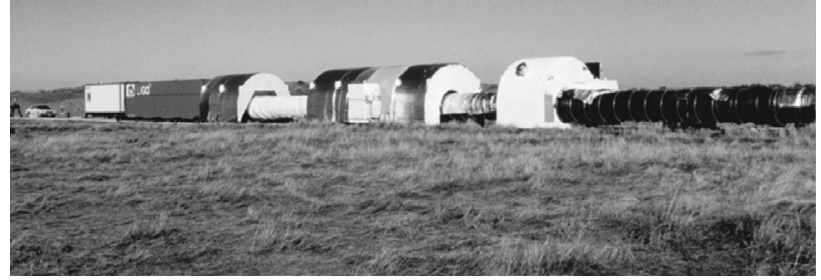

FIG. 2. Beam tube module assembly.

of the beam tube module assembly is provided in Fig. 2.

The beam tubes are passive components and, providing there are no leaks, the gas load is determined entirely by the outgassing of the material. The aim is to achieve the required pressures by end pumping only to minimize the pumping costs. The outgassing by water vapor and hydrocarbons is reduced by a factor of at least a 1000 by a $30 \mathrm{~d}$ bake at $168^{\circ} \mathrm{C}$. The outgassing of hydrogen is unaffected by the low temperature bake and is reduced by processing the stainless steel at $444{ }^{\circ} \mathrm{C}$ in a low humidity atmosphere for $36 \mathrm{~h}$ while still in the form of coils prior to fabricating the tube sections. Coupon tests were performed to establish the low hydrogen outgassing of each coil of steel used in fabricating the tube. The welding of the tube was carried out in a low humidity purge to reduce the reintroduction of hydrogen by the dissociation of water during the heating.

Maintaining a total leak rate of $1 \times 10^{-9} \mathrm{std} \mathrm{cc} / \mathrm{s}$ or less in a $2 \mathrm{~km}$ module was considered an extremely difficult task unless a systematic approach was taken to the leak testing. The decision was made to leak test each component, to better than $1 \times 10^{-10} \mathrm{std}$. cc/s, prior to installation into the beam tube module. A final leak test was then performed to confirm that the module in its entirety met the required leak rate. The $16 \mathrm{~km}$ of beam tube contained over $70 \mathrm{~km}$ of weld, divided into 800 tube sections, each needing to be leak tested. Leak tests were also required of the 808 field welded circumferential joints and the 72 ports and valves.

The leak testing of components had to be done in a timely manner to be economical and to prevent delaying the installation. It was essential that the component leak testing activities be operated on an efficient, production line basis.

\section{VISUAL INSPECTION AND REPAIR OF WELDING}

The initial weld quality test was a visual inspection by a weld supervisor of every inch of both weld passes, inside and out. The weld supervisors were highly trained in the art of automatic and manual welding and visual inspection of the finished weld. All questionable areas of the weld were rewelded prior to any leak test activity.

The rate of tube repairs or "pickups" detected by visual inspection slowly improved throughout the project. Some of the early tubes at Hanford had as many as 20-40 pickups while the Livingston job site produced 50 of its 400 tubes which were completely free of pickups. The majority of these pickups were small weld defects or areas of concern resulting from weld starts and stops or other welding abnormalities. 


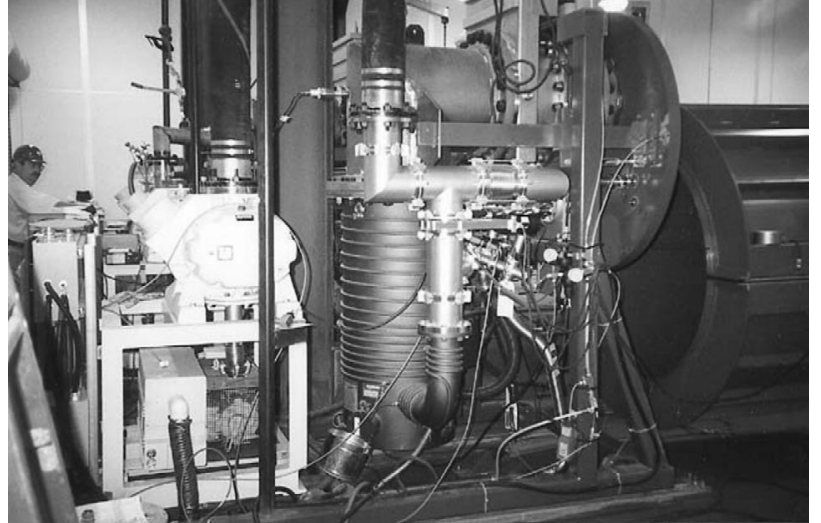

FIG. 3. Tube section leak test apparatus pumping systems.

\section{LEAK TESTING TUBE SECTIONS}

A sensitive leak test (less than $1 \times 10^{-10} \mathrm{std} \mathrm{cc} / \mathrm{s}$ of $\mathrm{He}$ ) was performed on each $20 \mathrm{~m}$ long tube section. The tube section leak test was accomplished in a separate leak detection room housing three identical test stations. The leak test was accomplished by machining the ends of the tube section (required for welding and alignment as well as leak testing) and sealing the tube between the two end heads. Seals were a butt gasket and an outer O-ring at each end, with a nitrogen purged annulus between. One of the end head assemblies contained the calibrated helium leak and the other end head assembly contained the pumping and leak detection systems. Prior to testing, a blind flange was installed on the tube sections having a $250 \mathrm{~mm}$ port.

The pumping of the tube was accomplished using a valved and $\mathrm{LN}_{2}$ trapped 20 in. diam diffusion pump backed by a rotary vane roughing pump. The helium mass spectrometer leak detector (HMS) was connected to the foreline of the diffusion pump such that either the main roughing pump, or a very small roughing pump, or the HMS could back the diffusion pump. A nonsilicon diffusion pump oil (Santovac $5^{\circledR}$ ) was chosen to permit easier cleaning of the tube if a backstreaming episode resulted from an equipment failure or operator error. See Fig. 3 for the tube section pumping system.

A clam shell type outer vessel enclosed the entire tube and was sealed to the tube at the ends of the tube section. In this manner, the tube section could be evacuated and the clam shell outer vessel, which completely surrounded the beam tube section, provided a space which could be flooded with helium to perform a "Hood" type HMS leak test. A view of the tube section leak test facility is provided in Fig. 4.

A major issue which drove the design of the tube section leak test apparatus was to prevent the buildup of helium background in the testing room which can migrate to the HMS via the pump exhaust system. The second issue was to be aware that leak testing and leak hunting are time consuming and costly. Therefore, everything that could be done to prevent leaks, the false indication of leaks, and helium satu-

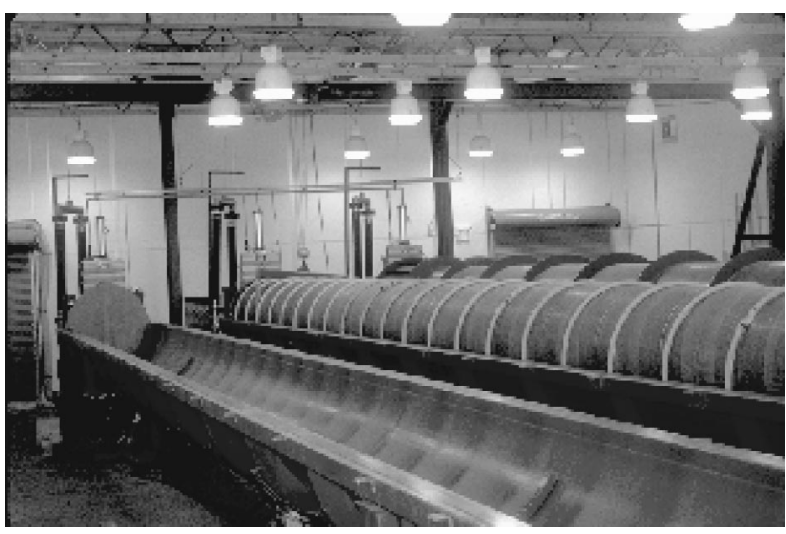

FIG. 4. Tube section leak test facility.

ration of the HMS units, was essential to the economic success of the project.

The clam shell outer vessel was sealed along all joints to prevent leakage of helium. It was provided with a roughing pump used first to evacuate the outer vessel prior to a backfill with helium and second (after the leak test), to evacuate the helium from the outer vessel and exhaust the helium outside of the building.

A separate HVAC system was installed in the tube section leak detection room. The HVAC system was fed with fresh air drawn from outside of the building to minimize the helium background within the room. The outside air was required due to the use of helium in the welding processes, which were performed in different sections of the fabrication building. In addition, a separate small room was erected adjacent to the leak detection room, housing the three HMS leak detectors. This room was also equipped with a separate HVAC system, with outside makeup air to minimize backstreaming of helium into the leak detectors.

The leak detector was equipped with a manual zero adjustment which was used to zero the indicated helium background within the test volume in order to be able to measure the sensitive helium leak rate within a reasonable amount of time. Testing indicated that helium backgrounds as high as the low to mid $10^{-8}$ std.cc/s of helium could be manually zeroed from the leak detector without effecting the sensitivity of the leak test. This was reconfirmed throughout the project by performing calibrations before and after every test.

Several steps were taken to ensure that accurate, reliable leak tests were performed. One of these steps was to locate a calibrated helium leak (low $10^{-10} \mathrm{std} . \mathrm{cc} / \mathrm{s}$ leak rate) on the opposite end of the tube from the pumping and leak detection systems. This allowed the measurement of true system sensitivity as opposed to machine sensitivity, which would be measured if the calibrated leak was placed directly on the HMS. With large pumping volumes or high helium backgrounds, the true system sensitivity would have been much less than the machine sensitivity. CB\&I has utilized this principal on all of their large volume leak testing projects for structures such as vacuum insulated spheres for the storage of various cryogens, space simulation chambers, a Tokamak 
ring, and a project that involved $1000 \mathrm{ft}$. of 24 in. diam high vacuum tube.

Another accuracy check was the use of an instrument to measure the concentration of helium near the bottom of the outer vessel. The instrument was a thermal conductivity gas analyzer calibrated specifically for helium by using a helium reference source. The measured value of the helium concentration was then used in the equation to determine the actual leak rate.

It was essential, in order to maintain a production line type leak testing program, to protect the HMS units from as much contamination as possible. A $\mathrm{LN}_{2}$ trap was provided on the inlet of the leak detectors to minimize contamination from the uncleaned beam tube sections (testing was performed prior to cleaning to eliminate the possibility of the cleaning water temporarily sealing a tiny leak). The leak detectors were also isolated from the pumping system for the majority of the time and connected to the system only immediately before the test. At all other times, the diffusion pump was backed by one of the roughing pumps.

The duration of a beam tube section test, including pumpdown of the beam tube section, could be as much as $8 \mathrm{~h}$ (or, more commonly, overnight pumpdown with about a $1 \mathrm{~h}$ test duration). It was therefore essential to the schedule that repeat tests were not required. A pretest and posttest calibration procedure was initiated in order to ensure that the accuracy of the test was confirmed and that failed equipment could be identified and moved offline and repaired.

The initial goal of the tube section leak test facility was to complete two tube section leak tests per day with the three leak test stations. The third tube section leak test station was envisioned to be utilized for leak hunting. Fortunately, no leaks were found in the beam tube sections and the third station was used continuously to increase the frequency of tube section tests. The tube section leak test facility was eventually capable of a maximum sustainable productivity of five tube section leak tests per day. This rate utilized a two man crew for one $10 \mathrm{~h}$ shift per day.

\section{VALVE AND PORT TESTS}

The welds of the $250 \mathrm{~mm}$ ports to the beam tube sections were checked during the tube section test. However, the high vacuum valve and its flange connection to the port were tested in a separate test. The valve and flange leak tightness was demonstrated by traditional bagging (Hood test) technique. The port was sealed by a custom made plug on the inboard end of the port where it attached to the beam tube. This plug sealed against the inside of the port nozzle neck and was provided with a calibrated leak and a helium feed connection. The pumping system and HMS were attached to a port in the blind flange on the outboard end of the valve. The leak test started with the valve open and the entire valve/ port assembly under vacuum from the inboard plug to the outboard blind flange. A pretest calibration was accomplished using the calibrated leak on the inboard plug. The flange connections and valve were leak checked by bagging the entire outside of the valve/port assembly and injecting

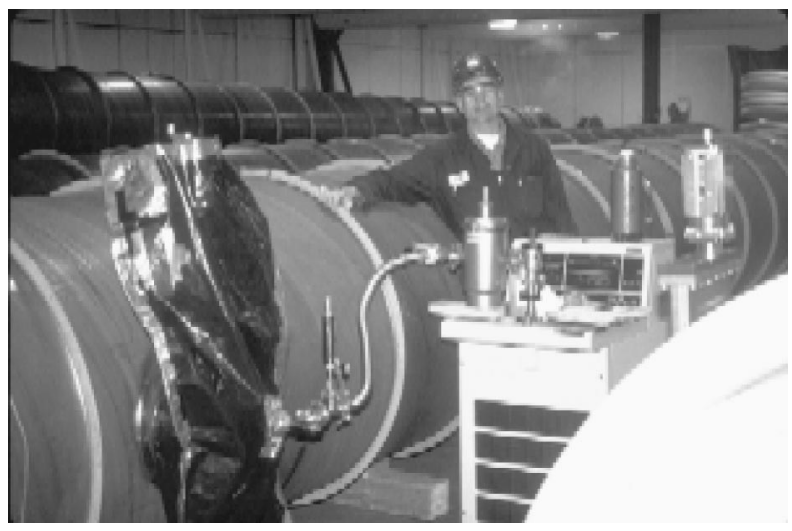

FIG. 5. Valve and port leak test apparatus.

helium into the bag. The valve was then closed and the inboard side of the valve was backfilled with helium through the plug helium connection. Using this technique, the valve was leak tested "the hard way" with vacuum trying to force the valve disk away from the sealing surface. This is the most severe method of leak testing a high vacuum valve. Helium concentration in the bag and behind the valve disk was calculated by measuring the oxygen concentration in the bag. Leak rate calculations were corrected for the helium concentration (see Fig. 5).

Direct measurement has shown that the helium will initially stratify in the top portion of the bag. It has been observed that during helium filling of the bag, the top portion of the bag may be near $100 \%$ helium while the bottom portion of the bag is below $1 \%$ helium. It is therefore essential to measure the helium concentration in the bottom portion of the bag, below any portion of the object to be tested.

\section{TUBE SECTION TO TUBE SECTION CIRCUMFERENTIAL WELD TESTS}

The last group of welds, which had to be tested prior to the final module leak test, were the circumferential welds between tube sections. As soon as a tube section was set in place during installation, it was provided with an initial alignment and then welded to the preceding tube section. The weld was then tested as a part of the tube section installation procedure.

The leak testing hardware consisted of two $180^{\circ}$ vacuum channels which were hooked together to cover the full $360^{\circ}$ circumferential weld. This vacuum channel was sealed to the outside of the beam tube and evacuated with a small turbomolecular pump and roughing pump combination. A $\mathrm{LN}_{2}$ trapped HMS was used, after isolation of the turbomolecular and roughing pump combination, during the leak test. An elastomeric ring was installed on the inside of the beam tube at the circumferential weld such that the space between the ring and the inside of the beam tube could be filled with helium. The leak test was a local, sensitive test and proved a leak rate of $1 \times 10^{-10}$ std. cc/s of helium or less. See Fig. 6 for a view of the leak test vacuum channel. 


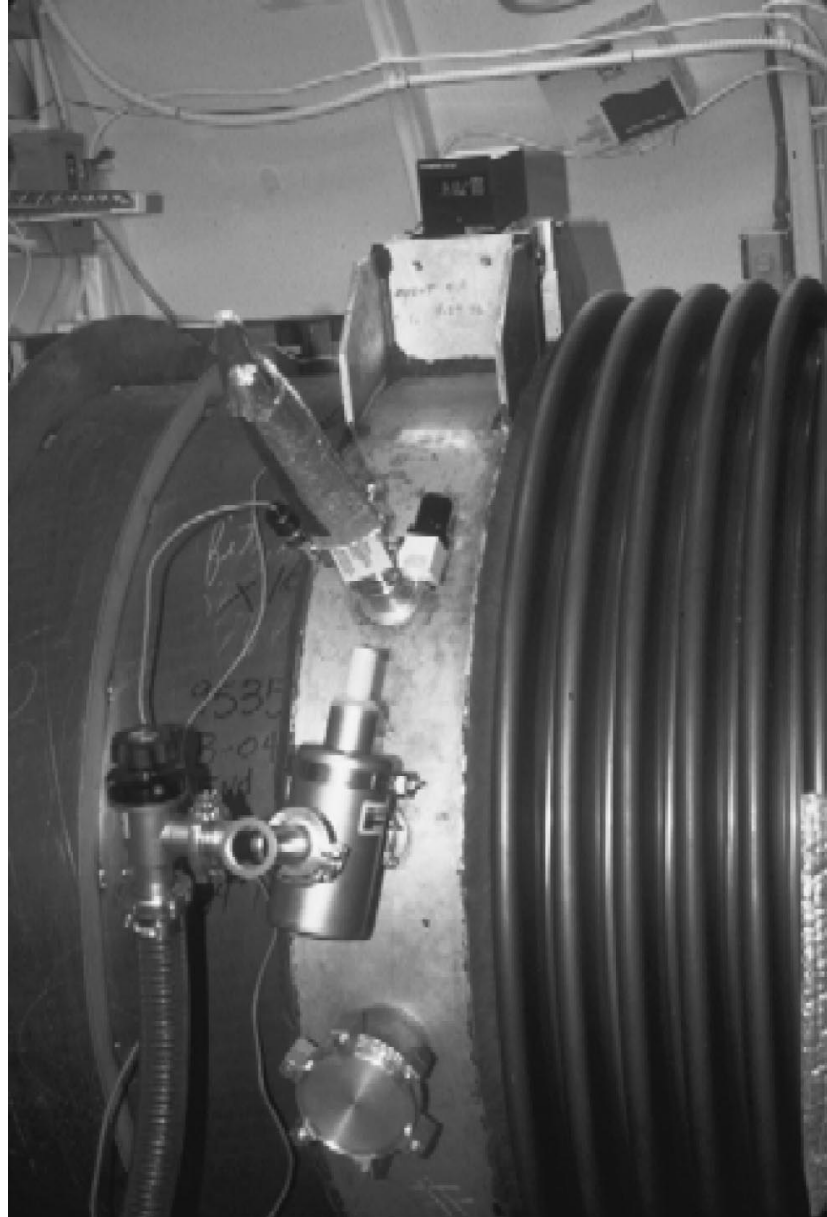

FIG. 6. Leak test channel for circumferential tube welds.

A number of different designs were tried for the vacuum channel and its seal to the outside of the beam tube. It was found that the simplest and most economical method was to use an electrical duct sealing putty to provide the seal between the vacuum channel and the beam tube. This manual installation of the "duct seal" putty was found to be quick, economical, and provided the sealing capabilities that a single elastomeric seal could not. Since the HMS was used only a short amount of time and was supplied with a $\mathrm{LN}_{2}$ trap, the HMS did not become contaminated by the oils in the putty.

\section{BEAM TUBE MODULE FINAL LEAK TEST}

The leak test of the entire $2 \mathrm{~km}$ beam tube modules would have been difficult and expensive using standard HMS techniques to prove a leak rate of $1 \times 10^{-9} \mathrm{std}$. cc/s of helium or less.

The method we adopted was to perform a leak assay by an accumulation in an entire module and analyze the residual gas spectrum for an air signature. The air signature is determined from the residual gas spectrum by solving for the amount of nitrogen, oxygen, and argon in a fit to a model for the gases represented in the tube. The cracking patterns for the significant gases in the tube were measured by calibration with those gases (i.e., $\mathrm{H}_{2}, \mathrm{NO}, \mathrm{CO}, \mathrm{CO}_{2}$, and $\mathrm{CH}_{4}$ ). The test sensitivity of the air signature was confirmed by direct injection of a known air leak into the tube during a separate accumulation test. A computer program was written to use the RGA output for 42 amu values to solve the overdetermined set of simultaneous equations for 20 model gases by $\chi^{2}$ minimization. The minimization was iterated by small variations in the cracking patterns for gases that had not been measured directly until an acceptable $\chi^{2}$ was obtained given the errors in the input RGA spectrum.

This approach was first tested in 1995 during CB\&I's qualification test project in which $140 \mathrm{ft}$. of the tube was assembled and tested by CB\&I at it's Plainfield, Illinois facility. The result of this test proved that the approach was reasonable and much more economical than a standard helium leak test program. The RGA used was equipped with a $90^{\circ}$ secondary electron multiplier and ion counter options to obtain improved sensitivity and accuracy.

The qualification test program in 1995 was evaluated by scaling from $140 \mathrm{ft}$. of tube to $2 \mathrm{~km}$ and it was determined that prior to module bakeout, an quantitative RGA leak test program would place upper bound limits of the beam tube module leak rate at $1 \times 10^{-8} \mathrm{std} . \mathrm{cc} / \mathrm{s}$ of air. After module bakeout, the RGA leak test program would place the upper bound limit of the leak rate at $1 \times 10^{-11} \mathrm{std}$. cc/s of air.

\section{BEAM TUBE MODULE FINAL LEAK HUNTING}

Leak hunting in a $2 \mathrm{~km}$ beam tube module would have been very difficult without a method of localizing the source of the leak to a small search area. Although never used, it was determined that a leak could be localized by utilizing a number of RGAs, which were spaced along the length of the beam tube module. The RGA calibration data were required to equalize the signals from each RGA. The source of the leak could then be localized by plotting the strength of the air signature indication at each RGA against its position along the tube length. It was estimated that a leak could have been localized, using nine RGAs along the length of the $2 \mathrm{~km}$ beam tube module, to a tube length of few meters for a leak as large as $10^{-6}$ Torr $\ell / \mathrm{s}$ or to within a $100 \mathrm{~m}$ for a $10^{-8}$ Torr $\ell / \mathrm{s}$ leak. The method used an algorithm to fit the model pressure profile for a leak by $\chi^{2}$ minimization to the air signature data at each RGA derived from the RGA spectra by methods similar to those described in the section on the leak assay. The method could have separated the location of three leaks of comparable size. After localization of the leak, standard detection methods would have been employed to find the precise location of the leak for subsequent repair.

\section{PROJECT RESULTS AND CONCLUSIONS}

The component leak testing facilities developed for this project worked well in a production line mode of operation. Component leak testing revealed a number of leaks in the high vacuum valves and a few $\mathrm{CF}$ type flange leaks due to assembly errors. However, of the 1680 sensitive component leak tests (typical sensitivities between mid $10^{-11}$ and mid $10^{-12} \mathrm{std} . \mathrm{cc} / \mathrm{s}$ of helium), there were no weld leaks. This 
was a zero failure rate in the $70+\mathrm{km}$ of weld produced for this project. The weld supervisors who inspected every inch of those $70+\mathrm{km}$ miles of weld caught every possible area of defective weld and performed repairs on the questionable welding prior to the leak tests. This accomplishment is a testimony to the quality of the visual inspection and is supported by the completed module leak tests.

A major reason for this success was that the welding technology and the weld procedures were well researched and tested prior to the start of production. Another significant reason is that the project staff viewed leak prevention as one of the key issues to the success or failure of the project. This was exhibited throughout the entire manufacturing and installation crew as a team objective, with dedication to a quality product.

CBI used helium sensors and oxygen sensors to measure the concentration of helium in its Hood tests. An oxygen sensor is an economical method of detecting a depletion of the air in a bag and may be readily available in many facilities, but it is not a specific measure of the helium concentration. It is recommended that where possible, direct helium concentration should be measured. CBI used portable oxygen sensors because of the limited availability of portable helium measuring instruments at the time of use. However, portable helium sensors are now available and CBI would recommend the use of direct helium sensors where possible.

As a result of outgassing from Viton O-rings in the 1120 $\mathrm{mm}$ module isolation valves and the $250 \mathrm{~mm}$ diagnostics valves, the leak rate of the completed modules could not be determined prior to the bakeout to a value better than approximately $3 \times 10^{-7} \mathrm{std}$. cc/s of air. The limit had been previously estimated at $1 \times 10^{-8} \mathrm{std} . \mathrm{cc} / \mathrm{s}$ of air for an all-metal system such as used in the qualification test. The reduction in sensitivity was due to the absorption and subsequent desorp- tion of air by Viton. The post bakeout leak rate, determined by the LIGO project team, for all modules baked out and leak tested to date is less than $1 \times 10^{-10} \mathrm{std} . \mathrm{cc} / \mathrm{s}$ of air.

The basic conclusions drawn from this project as well as the many years of experience developed by CB\&I is that it pays to spend money up front to prevent leaks and to ensure that the leak testing program is accurate and sensitive. The leak prevention activities include adequate study of the weld engineering and weld inspection either through visual or NDE techniques. The leak test program accuracy and sensitivity issues include control of helium background, control of the HMS contamination, measurement of the helium concentrations in a HMS test, and frequent measurement of test sensitivity. Leak hunting costs would be far in excess of normal leak prevention costs.

The other conclusion drawn from this project is that vacuum structures that are too large to be economically leak tested by traditional HMS techniques can be leak tested using residual gas analysis techniques.

\section{ACKNOWLEDGMENTS}

Chicago Bridge \& Iron Company thanks the LIGO project for the opportunity to work together on the LIGO beam tube modules. Members of the LIGO project and of CB\&I worked together to solve the many challenges faced during the $5 \frac{1}{2} \mathrm{yr}$ development, implementation, fabrication, and testing. LIGO is funded by the National Science Foundation, an agency of the United States Government, through a cooperative agreement issued to the California Institute of Technology (Caltech). Under this cooperative agreement, a subcontract has been executed between Caltech and the Massachusetts Institute of Technology providing for joint implementation of the LIGO project, LIGO project Document No. LIGO-P990023-00-B. 